\title{
Polarisation Measurement of THE EMitTed Thermal Radiation during LaSer Material Processing
}

\author{
Gerald Humenberger, Gerhard Liedl, Ferdinand Bammer \& Marco Ferrari
}
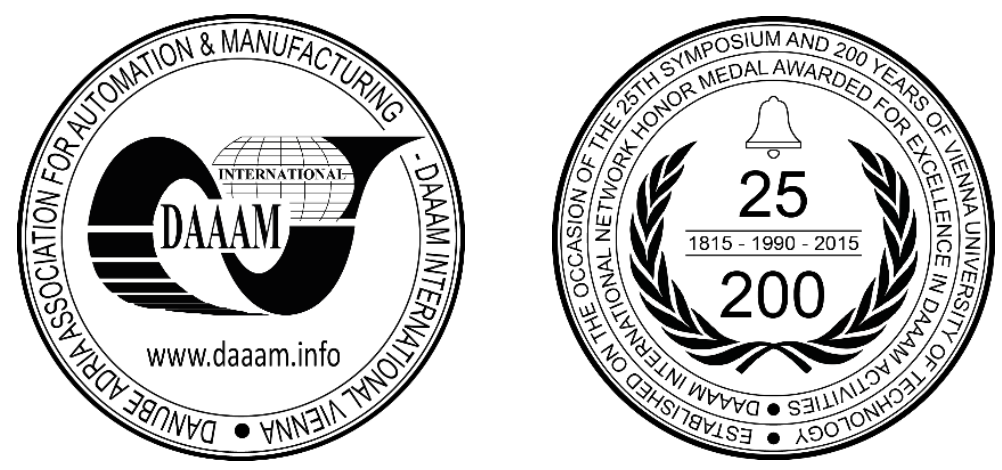

This Publication has to be referred as: Humenberger, G[erald]; Liedl, G[erhard]; Bammer, F[erdinand] \& Ferrari, M[arco] (2018). Polarisation Measurement of the Emitted Thermal Radiation During Laser Material Processing, Proceedings of the 29th DAAAM International Symposium, pp.0922-0930, B. Katalinic (Ed.), Published by DAAAM International, ISBN 978-3-902734-20-4, ISSN 1726-9679, Vienna, Austria

DOI: $10.2507 / 29$ th.daaam.proceedings. 133

\begin{abstract}
We present a new approach for quality control in laser material processing. By means of an active optical element (SCPEM - Single Crystal PhotoElastic Modulator) the emitted thermal radiation from the interaction zone of the focused laser radiation with the material surface is to be detected in order to determine a correlation to the process parameters from the polarisation state of the radiation. Due to the dependence of polarised radiation on the emission angle, the inclination of the interaction zone in the observed area can be deduced. For this purpose, an optomechanical setup for measuring two orthogonal polarisation directions of the emitted thermal radiation has been developed and a data acquisition system has been implemented. The amplitudes of two orthogonal polarisation directions could be determined from the signal modulated by the SCPEM during tests on different experimental setups. From the ratio of these polarisation components, emission angles can be derived using the Fresnel equations.
\end{abstract}

Keywords: laser material processing; laser cutting; thermal emission; SCPEM; polarisation

\section{Introduction}

Today, laser beam cutting is one of the standard methods in industrial manufacturing due to the high flexibility, excellent level of automation and the high quality of laser treated parts. New powerful brilliant laser sources allow very high cutting velocities and process monitoring devices face new challenges due to the process dynamics and the large number of parameters involved during laser material processing [1]. Different approaches in monitoring the quality of laser cuts have been evaluated by various groups [2], [3], [4]. A promising approach is the measurement of the emitted thermal radiation out of the cut kerf, with the opportunity to observe the laser cut coaxially to the optical path of the processing laser beam. During laser cutting, a narrow cut kerf develops with a characteristically inclined cutting front due to the energy balance [5]. The emitted thermal radiation from the inclined cutting front is polarised and can be described by Fresnel's equations. Such high processing speeds require an extremely fast detector. A measurement of two independent polarisation directions requires two independent sensors to detect both components simultaneously. An interesting alternative is to use a device that allows the detection of two independent polarisation states with only one detector. We will present such a device in the following part of the publication. 


\subsection{Polarisation of electromagnetic waves}

Thermal radiation can be described by means of electromagnetic waves. Besides their basic properties wavelength, frequency and amplitude such waves have another property called polarisation. The electric field vector of the emitted thermal radiation from the inclined cutting front oscillates only in one direction, this indicates that the radiation is linearly polarised. As seen in Fig. 1(a), we can separate the electrical field vector $\vec{E}$ of the linear polarised electromagnetic wave into a component parallel (p-polarised) and a component perpendicular (s-polarised) to the plane of incidence. P- and s-polarisation states are explained in Fig. 1(b).

(a)

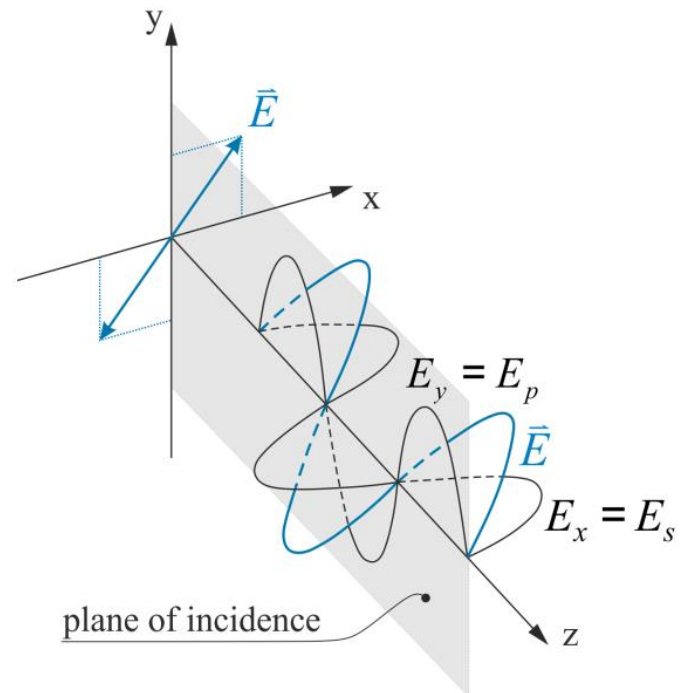

(b)

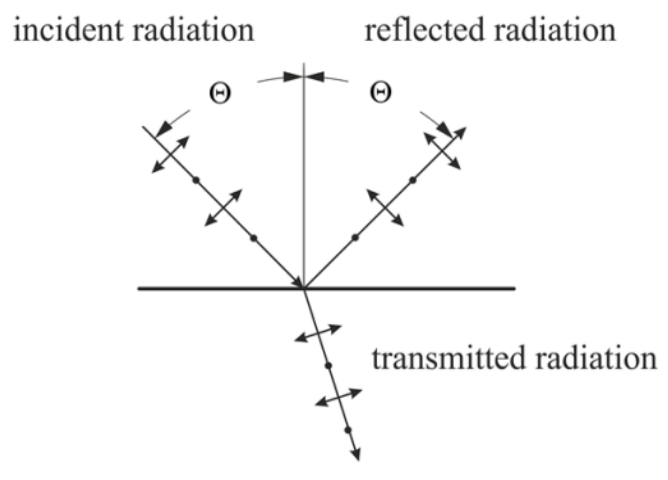

page $=$ plane of incidence

- s-polarisation, out of page

$\checkmark$ p-polarisation, parallel to page

Fig. 1. (a) Electric field vector [5] and (b) p- and s-states of polarisation

As we can see in Fig. 2(a), the p- and s-polarisation components show a strong dependence on the emission angle $\Theta$ (angle between surface normal and radiation direction), which can be described by the Fresnel's equations. The emissivity and the ratios of both polarisation directions have been calculated for iron and two different temperatures according to Fresnel's equations.

(a) Iron, $\lambda=10.6 \mu \mathrm{m}$

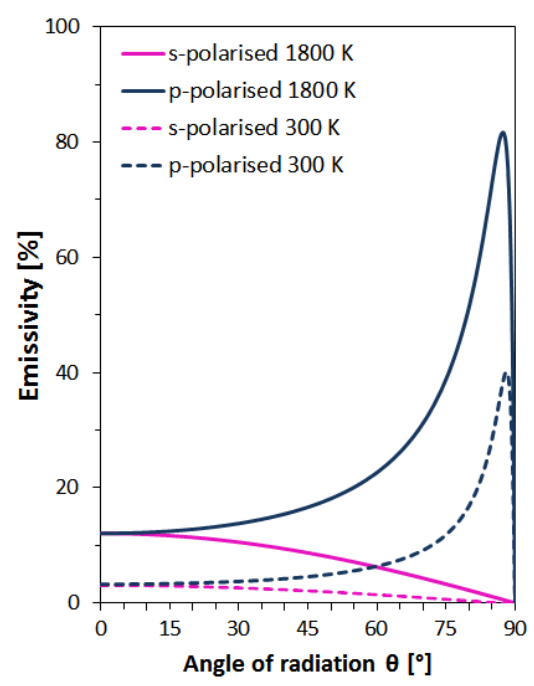

(b) Iron, $\lambda=10.6 \mu \mathrm{m}$

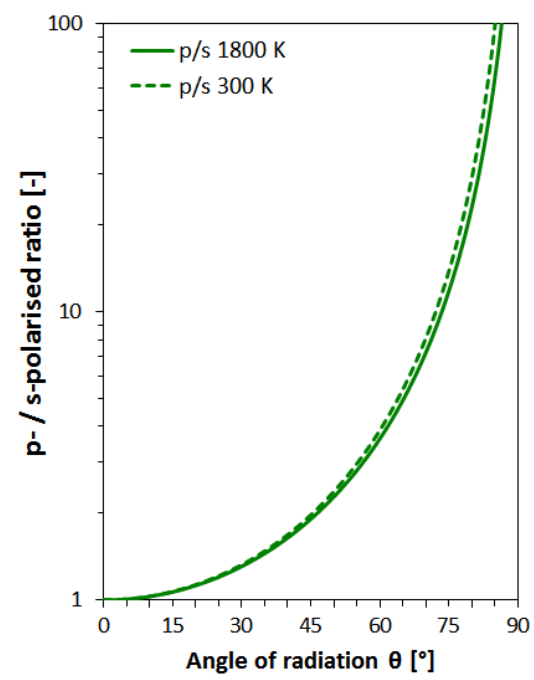

(c) Iron, $\lambda=10.6 \mu \mathrm{m}$

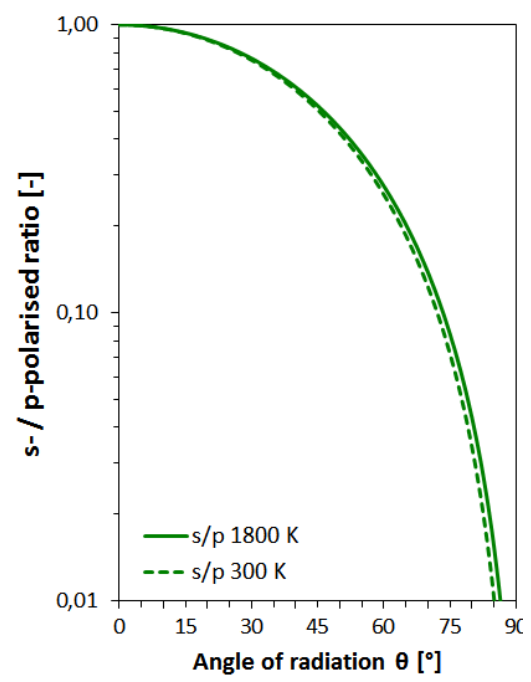

Fig. 2. (a) Influence of angle of radiation and polarisation state on emissivity at different temperatures and calculated ratio of p- to s-polarisation (b) and s- to p-polarisation (c) depending on the angle of radiation and temperature.

The temperature dependence of the emissivity can be avoided if we use the ratio between p- and s-polarised components as can be seen in Fig. 2(b). Diagram 2(b) and 2(c) show that the emission angle of the thermal radiation can be determined by measuring the $\mathrm{p}$ - and s-components of the polarisation, which in turn indicates the angle of inclination of the cutting front. In our first approach [6] we used a polarising beam splitter cube and two photodiodes to determine the two linear polarisation components related to the plane of incidence (see Fig. 3 ). 


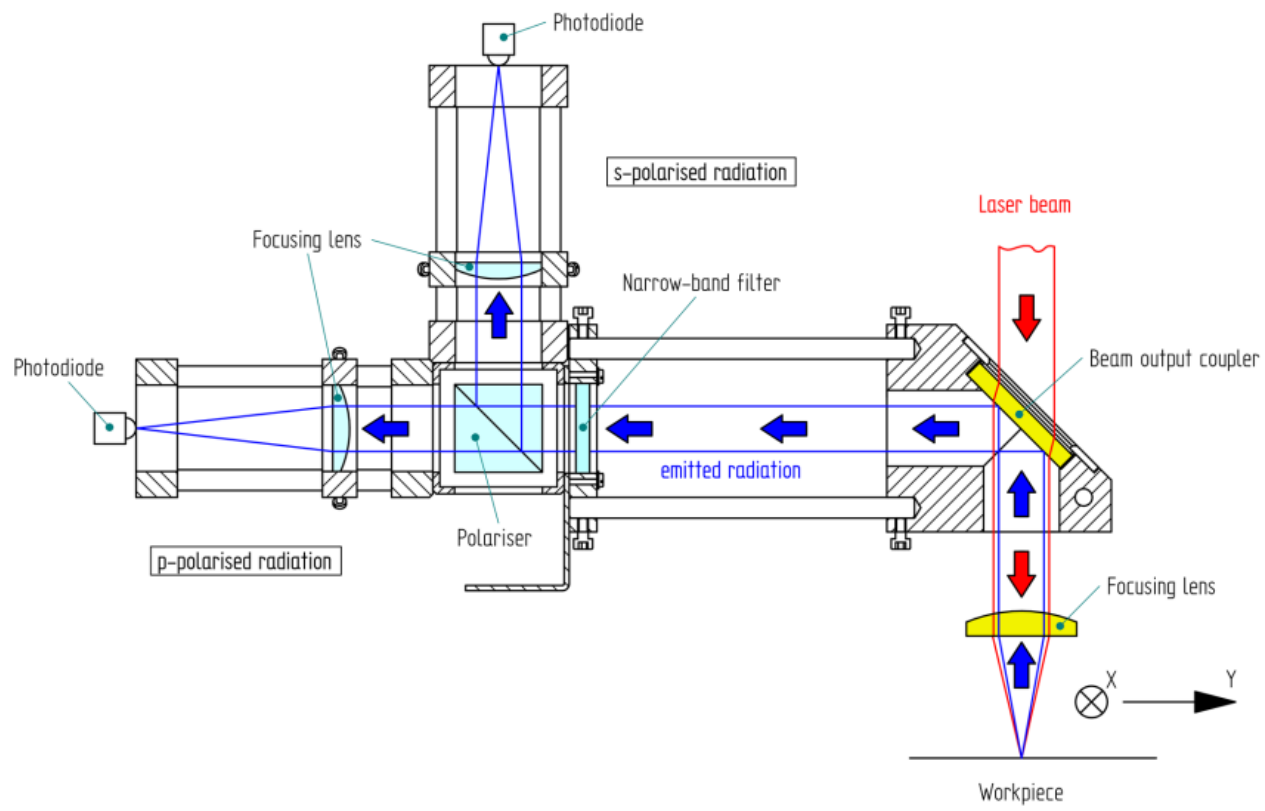

Fig. 3. Experimental setup with polarising beam splitter cube

With this setup it was possible to detect the emitted polarised thermal radiation during laser beam cutting of steel sheets. Fig. 4 shows measured polarisation components together with the calculated p- to s-ratio during laser fusion cutting of stainless steel with a thickness of $1.5 \mathrm{~mm}$ and $1500 \mathrm{~W}$ laser power. The sample has moved translational $50 \mathrm{~mm}$ in $\mathrm{x}$-direction, $10 \mathrm{~mm}$ in $\mathrm{y}$-direction and again $50 \mathrm{~mm}$ in $\mathrm{x}$-direction. At the regions were the cutting direction changes at $1000 \mathrm{~ms}$ and $1400 \mathrm{~ms}$, we can see a p- and s-polarisation interchange.

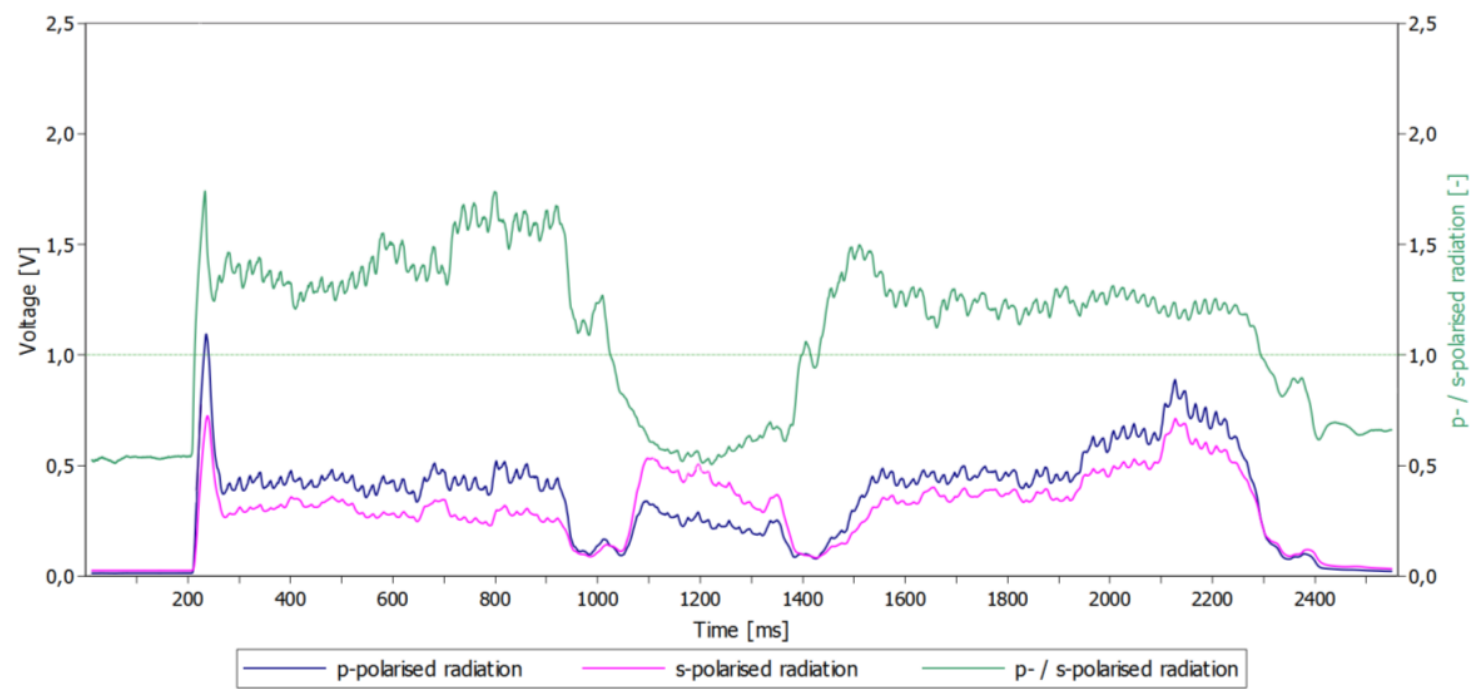

Fig. 4. Thermal emissions and calculated p- to s-ratio during laser fusion cutting of stainless steel with a thickness of $1.5 \mathrm{~mm}$ and $1500 \mathrm{~W}$ laser power with translational motion: $50 \mathrm{~mm}$ in $\mathrm{x}$-direction, $10 \mathrm{~mm}$ in y-direction and again 50 $\mathrm{mm}$ in $\mathrm{x}$-direction.

Nevertheless the calculated results of the p- to s-ratios and thus the determined angles of inclination were much lower as one would expect from the literature. From a comparison of the values of the ratio p- to s-polarised radiation with diagrams (b) and (c) in Fig. 2 one can assume that the angle of radiation varies between 40 up to 50 degrees. Known cutting front angles from the literature are somewhat greater, approximately 70 up to 89 degrees [5]. Probably our s-measurements are bigger than they should be which could be caused by a certain misalignment of the optical paths.

Since this setup defines a certain coordinate system, any change in the cutting direction has to be taken into account by a transformation of the accompanying coordinate system, which could only be done by integrating additional optical components to distinguish between changes in the emission characteristics of polarised components and directional changes. For this reason, we decided to take a different approach and chose an active optical component instead of the beam splitter cube, a so-called single crystal photoelastic modulator. 


\subsection{SCPEM}

A Single Crystal PhotoElastic Modulator (SCPEM) [7] modulates the polarisation of light due to the photoelastic effect. It is a piezoelectric optical transparent crystal that is electrically excited at one of its resonance frequencies. A configuration for the modulation of electromagnetic radiation can be seen in the sketch of Fig. 5 .

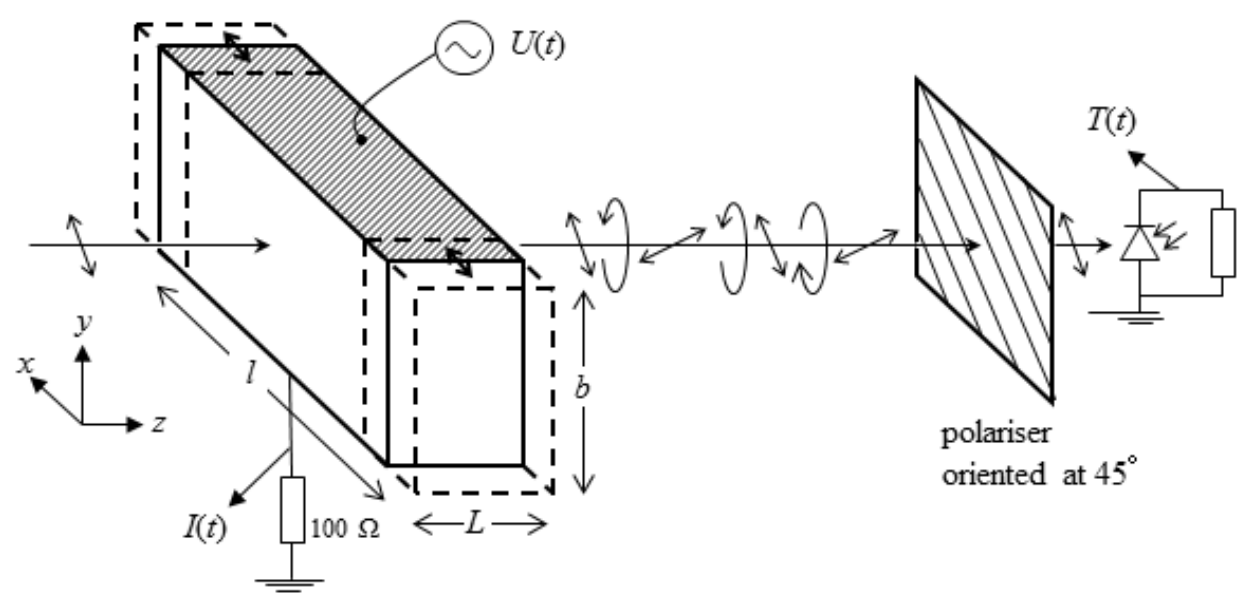

Fig. 5. Setup with a Single Crystal PhotoElastic Modulator (SCPEM) [7]

The SCPEM introduces a certain phase shift between to polarisation directions. By operating such a crystal at its resonance frequency, any incoming radiation is modulated and a periodic retardation occurs. The retardation can be described as

$\delta=\frac{2 \pi}{\lambda} L\left(n_{x}-n_{y}\right)$

where $L$ is the $\mathrm{z}$-dimension and $n_{x}, n_{y}$ are the driving voltage depended refractive indices for the $\mathrm{x}$ - and $\mathrm{y}$-direction (respectively $\mathrm{x}$ - and y-polarised radiation) of the crystal. The transmission $T(\delta)$ for the setup shown in Fig. 5 is

$T(\delta)=\cos ^{2}\left(\frac{\delta}{2}\right)=\frac{1+\cos (\delta)}{2}$

\section{Theoretical analysis}

For the following illustrations, the Stokes parameter approach is used in conjunction with Mueller matrices to represent the polarised radiation. One of the advantages of these parameters is that they can be calculated by simple measurements of the intensity after passing through different optical components and thus the polarisation state can be calculated. Another advantage is the possibility to describe partial polarised and unpolarised radiation, so we can describe by the use of Stokes parameters all types of polarisation. In our case, the influences of the different optical components on the radiation along the optical path in $z$ direction (see Fig. 5) can be calculated in conjunction with the Mueller calculus.

The state of polarisation of an electromagnetic wave can be expressed by the four Stokes parameters $S_{0}, S_{1}, S_{2}$ and $\mathrm{S}_{3}$. They are often combined into the four-dimensional Stokes Vector:

$$
\vec{S}=\left(\begin{array}{l}
S_{0} \\
S_{1} \\
S_{2} \\
S_{3}
\end{array}\right)=\left(\begin{array}{l}
\left\langle E_{x}^{2}\right\rangle+\left\langle E_{y}^{2}\right\rangle \\
\left\langle E_{x}^{2}\right\rangle-\left\langle E_{y}^{2}\right\rangle \\
2\left\langle E_{x} E_{y} \cos (\rho)\right\rangle \\
2\left\langle E_{x} E_{y} \sin (\rho)\right\rangle
\end{array}\right)=\left(\begin{array}{l}
I_{x}+I_{y} \\
I_{x}-I_{y} \\
I_{+45^{\circ}}-I_{-45^{\circ}} \\
I_{R}-I_{L}
\end{array}\right)
$$

The terms in brackets \langle\rangle represents time-averaged values and $\rho$ is the relative phase between the field vectors $E_{x}$ and $E_{y}$. $S_{0}$ represents the total radiation intensity and the parameters $S_{1}, S_{2}$ and $S_{3}$ represent components of the Stokes vector related to linear and circular polarised components shown in Fig. 6. $I_{R}$ and $I_{L}$ represents the radiation intensity of rightcircular and left-circular polarised fractions. 

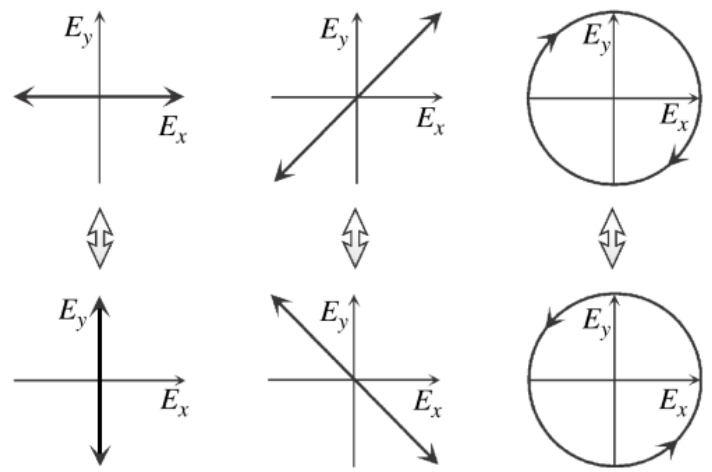

(a) $S_{1}=I_{x}-I_{y}$

(b) $S_{2}=I_{+45^{\circ}}-I_{-45^{\circ}}$

(c) $S_{3}=I_{\mathrm{R}}-I_{\mathrm{L}}$

Fig. 6. Stokes parameters $S_{1}, S_{2}$ and $S_{3}$ based on radiation intensity [8]

The Mueller calculus is a matrix method (transformation matrix) to manipulate the Stokes parameters. The Mueller matrix for the SCPEM is expressed in equation (4):

$$
M_{\text {SCPEM }}=\left[\begin{array}{cccc}
1 & 0 & 0 & 0 \\
0 & 1 & 0 & 0 \\
0 & 0 & \cos (\delta) & -\sin (\delta) \\
0 & 0 & \sin (\delta) & \cos (\delta)
\end{array}\right]
$$

$\delta$ is the phase difference relative to the $x$ and $y$ directions due to the photoelasticity of the crystal, which is modulated due to driving circuit at the resonance frequency of the crystal. [9]:

$$
\delta=A \sin (\omega t)
$$

With the relative phase amplitude $A . \omega=2 \pi v$ and $v$ is the resonance frequency of the crystal. The next optical element in the path of infrared radiation that influences the state of polarisation is the analyser:

$$
M_{\text {Analyser }}=\left[\begin{array}{cccc}
1 & \cos (2 \alpha) & \sin (2 \alpha) & 0 \\
\cos (2 \alpha) & \cos ^{2}(2 \alpha) & \sin (2 \alpha) * \cos (2 \alpha) & 0 \\
\sin (2 \alpha) & \sin (2 \alpha) * \cos (2 \alpha) & \sin ^{2}(2 \alpha) & 0 \\
0 & 0 & 0 & 0
\end{array}\right]
$$

$\alpha$ represents the rotation angle between the $x$ direction and the orientation of the analyser as can be seen in Fig. 5 . We established intentionally a certain ratio between the s- and p-polarised components of the emitted thermal radiation by using a linear polariser in front of the SCPEM (see Fig. 7). That setup leads to only linear polarised $\vec{S}_{I n}$. Applying the Mueller calculus on the Stokes vector describing the incoming radiation leads to:

$$
\vec{S}_{\text {Out }}=M_{\text {Analyser }} \cdot M_{\text {SCPEM }} \cdot \vec{S}_{\text {In }}
$$

$\vec{S}_{\text {Out }} \ldots$ Polarisation state of the resulting Stokes vector behind the analyser

$\vec{S}_{I n}$... Polarisation state of the source of radiation, in our setup from the linear polariser $\left(\vec{S}_{I n}=\vec{S}_{L P}\right)$

With our experimental setup only the first row of the matrix multiplication can be determined:

$$
S_{\text {Out } 0}=S_{I n 0}+\cos (2 \alpha) * S_{I n 1}+\sin (2 \alpha) * \cos (\delta) * S_{I n 2}-\sin (2 \alpha) * \sin (\delta) * S_{I n 3}
$$

Now we can define the following boundary conditions:

- $S_{I n 3}=0 \ldots$ no circular polarised radiation due to the use of a linear polariser

- $\delta_{\max }=\pi \ldots$ SCPEM acts as a Half-wave plate $(\lambda / 2$ - retarder $)$ 
After some transformations and substitutions a term results for the ratio of the electrical field vector components which can be expressed as the angle of rotation of the linear polariser in respect to the $\mathrm{x}$-axis:

$$
\gamma_{I n}=\arctan \left(\frac{E_{y}}{E_{x}}\right)=\arctan \left(\frac{s * Q \pm \sqrt{c^{2}+s^{2} * Q^{2}-1}}{1-c}\right)
$$

With $c=\cos (2 \omega) ; s=\sin (2 \omega)$ and $Q=\frac{I_{0}+I_{\pi}}{I_{0}-I_{\pi}}$

As the theoretical treatment shows, it is possible to determine the components of linear polarised radiation by replacing to individual polarisation detectors by a combination of a SCPEM and a linear polariser. As a consequence, we are now able to modify our measurement setup and skip the two optical paths of the detector used till now.

\section{Experimental setup}

\subsection{Optomechanical design}

A sketch of the new setup based on the theoretical analysis is shown below.

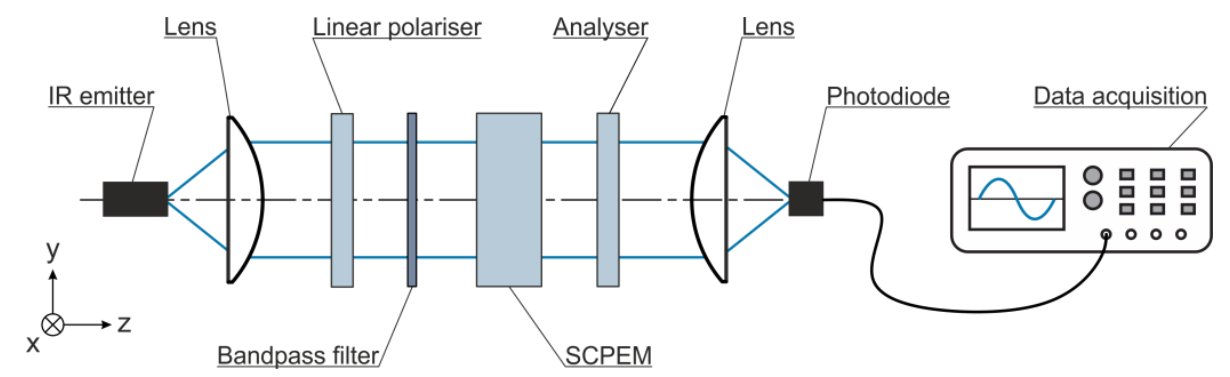

Fig. 7. Scheme of the experimental setup to determine the rotation angle of the polariser

Before we could use that new setup during laser beam cutting, we have to test its feasibility. The sketch in Fig. 7 shows the experimental setup for measuring the rotation angle of an adjustable linear polariser in respect to the $\mathrm{x}$-axis. The IR emitter emits thermal radiation which is collimated by a lens and polarised by a linear polariser. Afterwards the radiation is partially transmitted by a bandpass filter and will be modulated from the SCPEM in combination with the analyser. The modulated electromagnetic waves are then focused with a lens onto the active area of a photodiode. A transimpedance amplifier together with a data acquisition system has been used for recording signals during the test runs. The photo in Fig. 8 shows an experimental setup used for feasibility tests of the SCPEM-based detector system. In this setup, the reflected radiation of the IR emitter is measured instead of the radiation by a linear polariser as in Fig. 7.

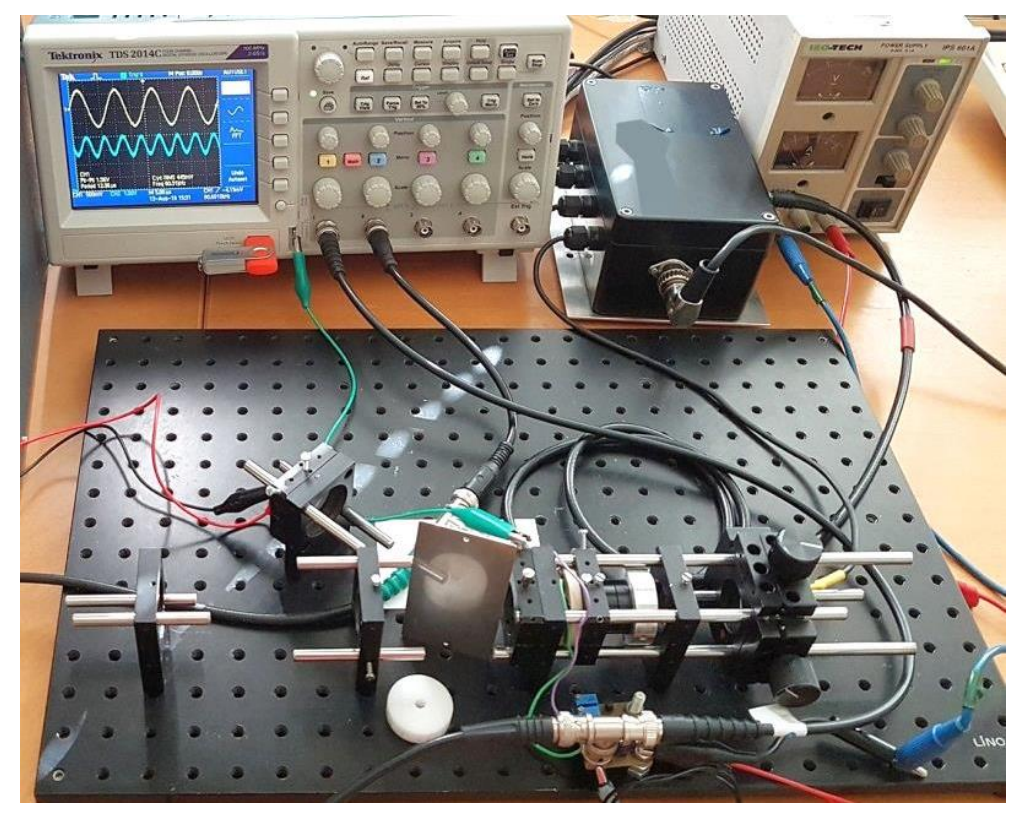

Fig. 8. An experimental setup used for feasibility tests of the SCPEM-based detector system. 


\subsection{Optical and electronic components}

By the use of a dielectric bandpass filter the optical system has been optimised for a wavelength of $1500 \mathrm{~nm}$ to eliminate the influence of reflected or scattered laser radiation and to avoid any other radiation which could disturb the measurements. A further advantage is the elimination of the wavelength dependence of the SCPEM (Fig. 9(a)). Antireflection coatings on the linear polarisers and lenses reduce transmission losses to a minimum.

(a)

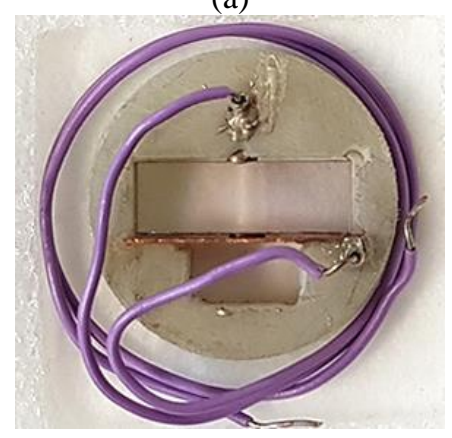

(b)

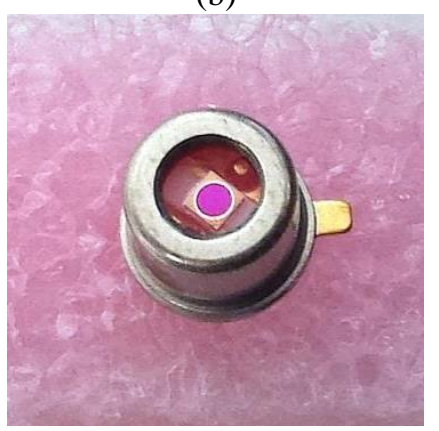

(c)

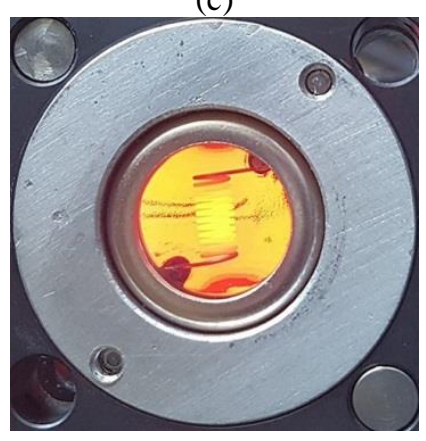

Fig. 9. (a) SCPEM crystal in plastic housing; (b) SWIR photodiode for infrared radiation; (c) IR emitter

A so-called SWIR photodiode (Short-wave infrared, Fig. 9(b)) is used for the detection of the infrared radiation with a wavelength of $1500 \mathrm{~nm}$. The SCPEM is implemented into an optical mounting system. For an operation of the crystal at its resonance frequency, an adapted electronic circuit has been designed and built. Electrodes for the electrical excitation of the crystal have been attached to both xz-surfaces of the SCPEM (see Fig. 5) and connected to the driving circuit.

\subsection{Data Acquisition}

For data acquisition we used an open-source measurement and control device based on STEMlab from Red Pitaya (see Fig. 10). It is equipped with a C-code programmable FPGA (field-programmable gate array) and an ADC which can digitise an analogue signal with a maximum sampling rate of $125 \mathrm{MS} / \mathrm{s}$ in 14 bit. The correlation shown in formula (9) has been programmed in Matlab and subsequently implemented as ported C-code in the FPGA. All relevant data are extracted from the digitised signal so that the angular position of the linear polariser can be calculated in relation to the X-axis. The programming language Python has been used to develop an interface to communicate with the STEMlab via Ethernet and for visualisation purposes (GUI) (see Fig. 11) on a personal computer or Laptop.

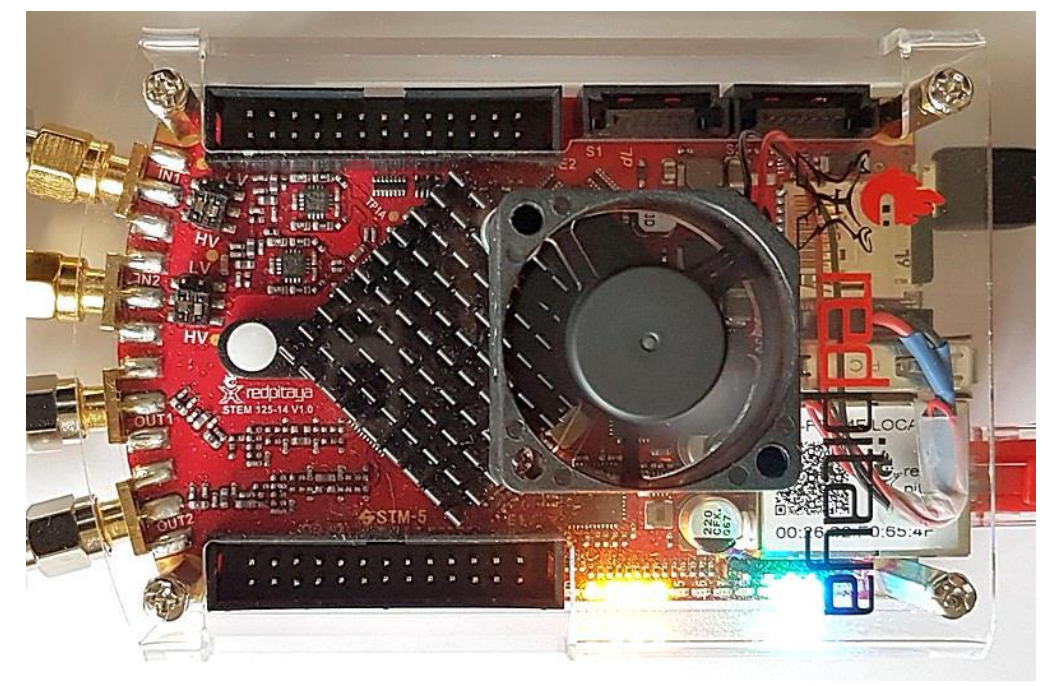

Fig. 10. Open-source measurement and control device STEMlab from Red Pitaya

Using the latest version of the graphical user interface, the connection between the STEMlab and the PC can be directly initiated and a measurement can be started, paused or stopped. Current data of the SCPEM, such as the oscillation frequency of the crystal or the applied retardation, can be displayed as well as specified for new measurements. The currently determined values of the angle measurement are displayed online and are also continuously displayed in a diagram. 


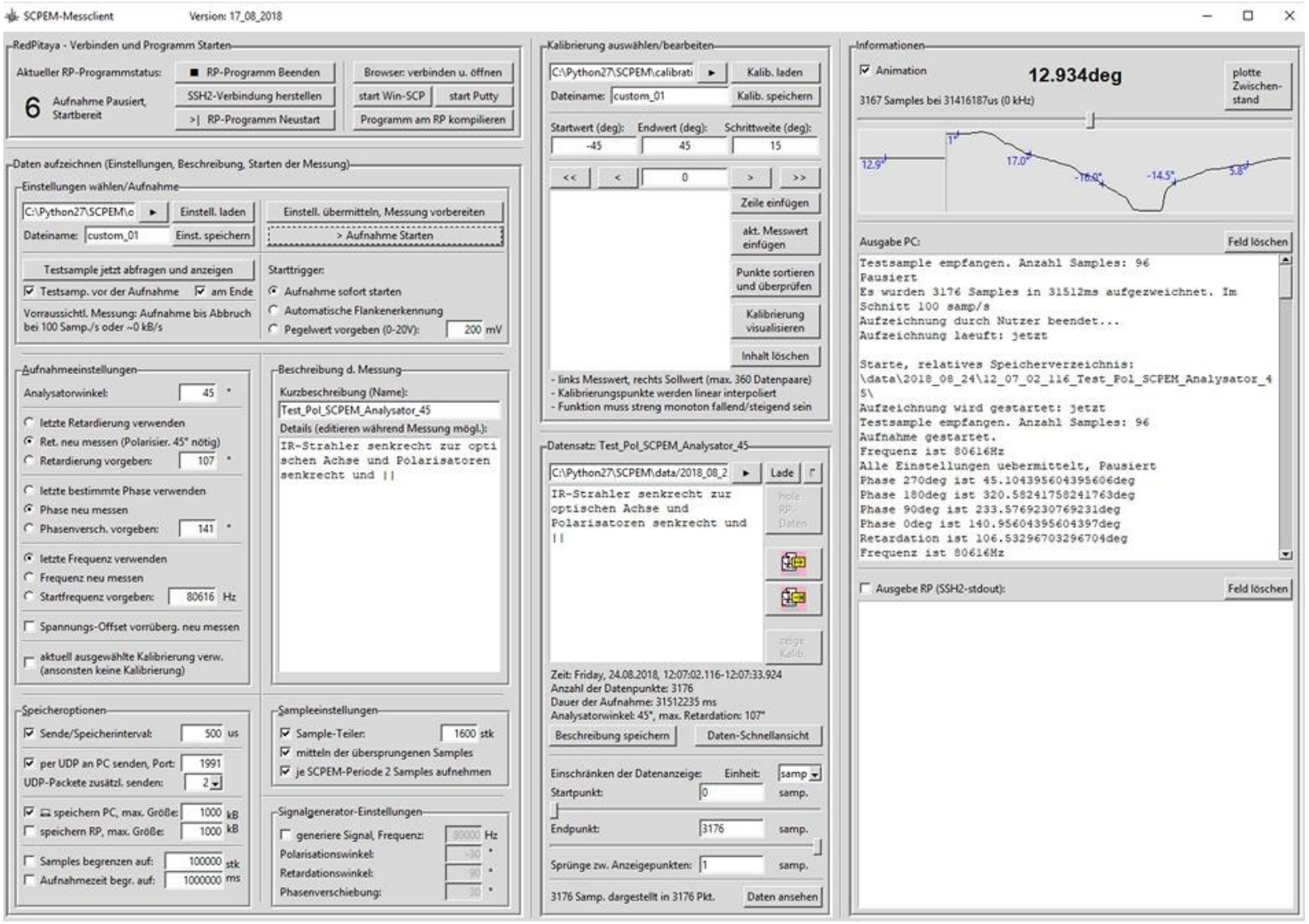

Fig. 11. Current state of the graphical user interface (GUI) for data acquisition

\section{Results and discussion}

During the initial testing, visible light sources such as LEDs and laser diodes have been used for easier alignment and the availability of cheap optical components and sensors. At the beginning, the focus was on the design of the control system for the SCPEM and an amplifier circuit for the photodiode. Both electrical circuits were initially developed using breadboards and only realised in PCBs after functional tests. In the first experimental setups, an attempt was made to specify a corresponding polarisation ratio with two beam sources representing the two radiation intensities $I_{x}$ and $I_{y}$. This intentionally established ratio due to different changes in the two intensities of the sources by means of grey filters was modulated by the SCPEM and the analyser and recorded. The corresponding intensity ratios have been extracted from the signals and then compared with the intentionally set values. In the experiments with visible radiation, a correlation with the intentionally set angles of the polariser could only be verified in a very narrow angle range up to approx. $20^{\circ}$. Throughout these experiments, the analyser was always set at $45^{\circ}$ in relation to the $\mathrm{x}$-axis of the SCPEM which influences the sinusoidal progress of the signal course. Although the general feasibility could be demonstrated, several ambiguities remained and we decided to use a different approach for the verification of the functionality of our measurement setup.

It is the experimental setup as shown in Fig. 8 optimised for the wavelength of $1500 \mathrm{~nm}$. With this configuration only one beam source is needed and by using a linear polariser the polarisation direction can be adjusted very precisely. Due to the different wavelength, we had to use a photodiode for the infrared wavelength range, as shown in Fig. 9(b). In addition, it was necessary to modify the amplifier circuit to suit the requirements of the SWIR photodiode and the driving circuit of the SCPEM has also been further optimised. As a new radiation source we used an IR emitter shown in Fig. 9(c) and the dielectric bandpass filter as described in subsection 3.2 on page 7. After adaptations of the optomechanical setup and optimisations of the data acquisition system, measurements could be carried out successfully. During these measurements, the intentionally set angular positions of the linear polariser could be detected with sufficient accuracy. Furthermore, the previously mentioned problem of the small measurable angle range was also investigated. For this purpose, the angle of the analyser in relation to the SCPEM has to be varied at a constant rotation angle of the linear polariser. This required an additional variable in the calculation and thus a further adaptation of the data acquisition system. As a result, measurements with different positions of the analyser in relation to the SCPEM have been performed. The results are shown in Fig. 12. As we can see from the diagram, the measurement range increases with a smaller angular position of the analyser. For an angle of $10^{\circ}$ between the analyser and the SCPEM, the deviation between measured and real values remains below $2^{\circ}$ for an angular range of $\pm 70^{\circ}$. However, when using a larger measuring range, the error in determining the angular position of the linear polariser increases as well. 


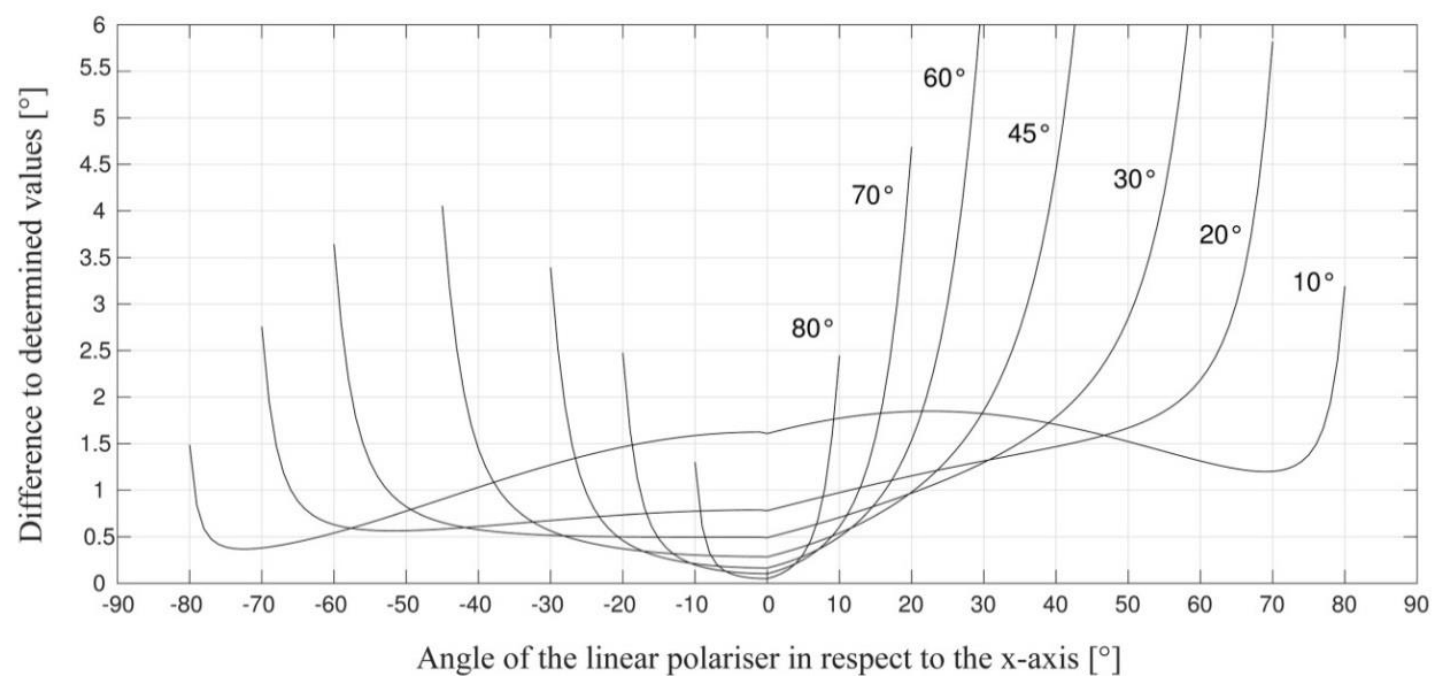

Fig. 12. Difference of the determined angles to the intentionally set angles of the linear polariser for different positions of the analyser [10]

\section{Conclusions and outlook}

The experiments carried out show that the use of a photoelastic modulator can detect linearly polarised radiation with a high measuring frequency and determine the direction of the oscillating electric field vector in a large angular range. This makes it possible to provide a very fast polarisation measurement at high speeds during laser material processing. For such applications, further in-depth investigations are necessary. First of all, it is required to verify the measuring principle for the emission of polarised thermal radiation from heated inclined surfaces in order to be able to use the system for investigations in material processing, such as laser beam cutting. The measuring system must also be adapted to the intensities of the radiation emitted by the observed processing area during laser material processing. This includes the amplifier circuit for the photodiode and most likely the use of a lock-in amplifier to optimise the signal-tonoise ratio. In order to make the measuring system capable for industrial use, directionality needs to be eliminated as well, since laser material processing is generally used for complex geometries.

\section{Acknowledgments}

The authors would like to thank for the support of this project carried out within the framework of the funding program Sparkling Science, funded by the Federal Ministry of Education, Science and Research.

\section{References}

[1] Begic-Hajdarevic, D.; Pasic, M.; Cekic, A. \& Mehmedovic, M. (2016). Optimization of process parameters for cut quality in $\mathrm{CO} 2$ laser cutting using taguchi method, Proceedings of the 19th International DAAAM Symposium, Published by DAAAM International, Vienna, DOI: 10.2507/27th.daaam.proceedings.024

[2] Dubrov, A. V.; Dubrov, V. D.; Zavalov, Y. N.; Makarova, E. S. \& Dubrovin, N. G. (2012). Pyrometry diagnostic in laser cutting technology, Available from: https://doi.org/10.1117/12.923016: 2018-10-01

[3] Adelmann, B.; Schleier, M.; Neumeier, B.; Wilmann E. \& Hellmann, R. (2015). Optical cutting interruption sensor for fiber lasers, Available from: http://dx.doi.org/10.3390/app5030544: 2018-10-01

[4] Molitor, T.; Schneider, F.; Bußkamp, T.; Thombansen, U.; Eppelt, U.; Petring, D. \& Schulz, W. (2010). Selfoptimizing focus technique (SOFT) - A new approach on the way to next generation cognitive laser cutting machines, Proceedings of ICALEO 2010, ISBN 978-0-912035-61-1, pp. 233-238, LIA, Orlando

[5] Hügel, H. \& Graf, T. (2009). Laser in der Fertigung, Vieweg + Teubner GWV Fachverlag, 978-3-8348-9570-7, Wiesbaden

[6] Humenberger, G.; Liedl, G. \& Heidlmayr S. (2014). Measurement of the emitted thermal radiation during CO2laser cutting, Proceedings of MOTSP 2014, Croatia, ISSN 1848-5022, Croatian Association for PLM,

[7] Bammer, F. (2009). Single Crystal Photo-Elastic Modulators, In: High-Power and Femtosecond Lasers, Barret, P.H., Palmer, M., 71-123, Nova Science Publishers, Inc., ISBN 978-1-60741-009-6, New York

[8] Fujiwara, H., (2007). Spectroscopic Ellipsometry: Principles and Applications, John Wiley \& Sons, Ltd., ISBN 978-0-470-01608-4, Chichester

[9] Jasperson, S. N. \& Schnatterly, S. E. (2003). An improved method for high reflectivity ellipsometry Based on a new polarization modulation technique, Available from: https://doi.org/10.1063/1.1684062: 2018-10-01

[10] Ferrari, M. (2018). Aufbau und Test eines Messsystems zur Detektion thermischer Strahlung während der Lasermaterialbearbeitung, Master thesis, Vienna University of Technology, Vienna, Austria, unpublished 